\title{
A Simple Halogen-free Magnesium Electrolyte for Reversible Magnesium Deposition through Co-solvent Assistance
}

Shengqi Fan, ${ }^{a €}$ Genevieve M. Asselin, ${ }^{a €}$ Baofei Pan, ${ }^{b}$ Hao Wang, ${ }^{b}$ Yang Ren, ${ }^{c}$ John T. Vaughey, ${ }^{b}$ Niya Sa*a

*a University of Massachusetts, Boston, MA 02125

${ }^{b}$ Chemical Sciences and Engineering Division, Argonne National Laboratory, Lemont, IL 60439

'Advanced Photon Source, Argonne National Laboratory, Lemont, IL 60439

$€$ Equally contributing authors

\section{Electrochemical Stability of Current Collectors:}

Oxidation limit of a $0.5 \mathrm{M} \mathrm{Mg}(\mathrm{TFSI})_{2}$ in DMA-THF electrolyte are tested against stainless steel (Figure S1a), Cu (Figure S1b), Mo (Figure S1C), and Al (Figure S1d) substrates. Results suggest the electrolyte stability for the tested current collectors is close or beyond $\sim 3.0 \mathrm{~V}$ versus a $\mathrm{Mg}$ metal reference electrode, indicating the less corrosive nature of the electrolyte possibly due to the weak interaction between the TFSI anion and the Mg.
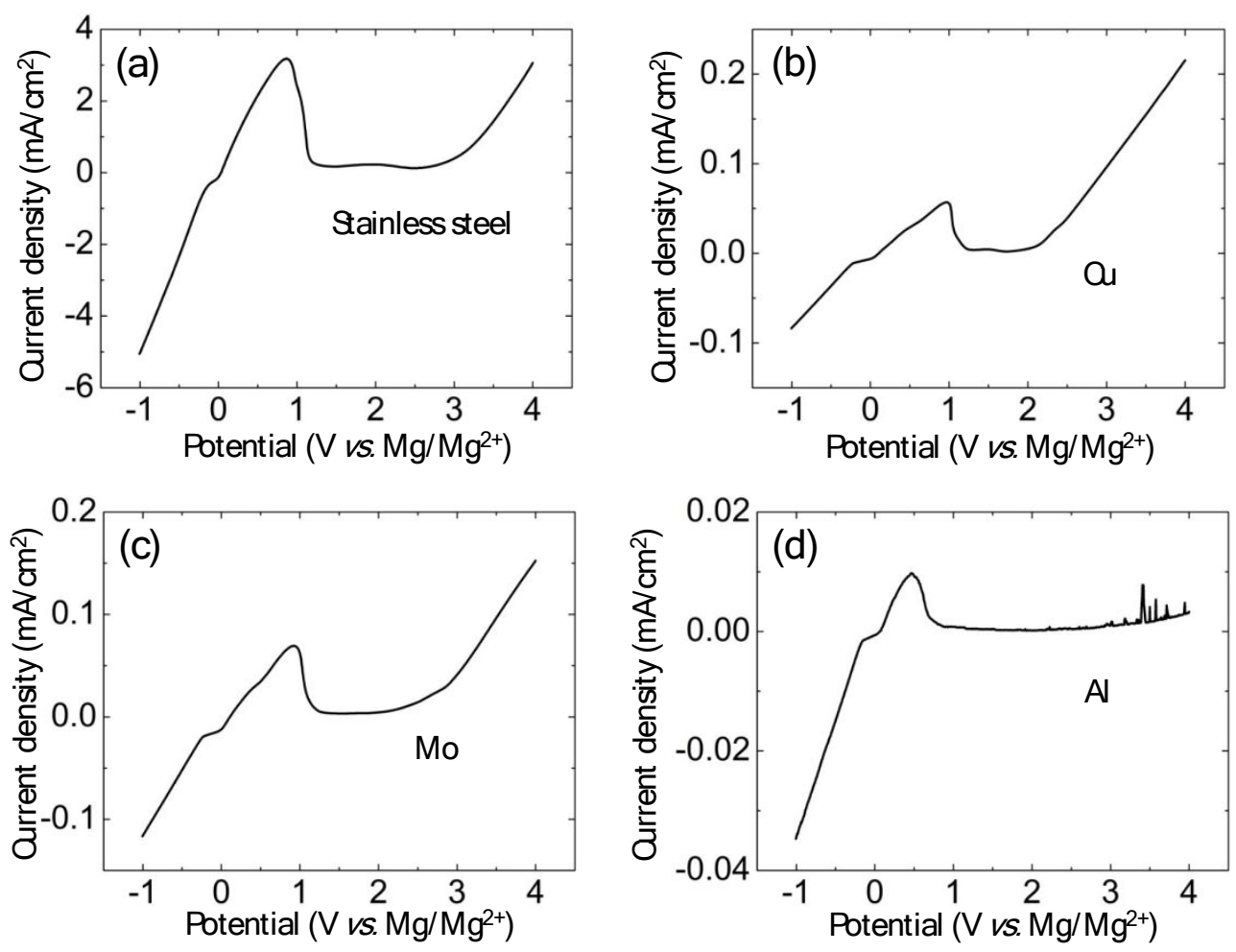

Figure S1. Electrochemical stability of the $\mathrm{Mg}(\mathrm{TFSI})_{2}(\mathrm{THF})_{4}$ electrolyte on stainless steel (panel a), Cu (panel b), Mo (panel c) and Al (panel d) current collectors. Linear Scan Voltammetry of a $0.5 \mathrm{M} \mathrm{Mg}(\mathrm{TFSI})_{2}$ in DMA-THF electrolyte on current collectors at a scan rate of $50 \mathrm{mV} / \mathrm{sec}$. 


\section{Electrochemical properties of recrystallized $\mathrm{Mg}(\mathrm{TFSI})_{2}$ in DMA-THF electrolyte:}

Figure S2 and S3 present the corresponding cyclic voltammetry of a $0.5 \mathrm{M} \mathrm{Mg}(\mathrm{TFSI})_{2}$ in DMA-THF electrolyte upon $1^{\text {st }}$ and $2^{\text {nd }}$ recrystallization. Cyclic voltammetry with clear reversible $\mathrm{Mg}$ deposition were presented even after two times of recrystallization, suggesting the $\mathrm{Mg}(\mathrm{TFSI})_{2}(\mathrm{THF})_{4}$ is the active component contributing to the electrochemical activity for reversible $\mathrm{Mg}$ deposition. Table $\mathbf{S 1}$ lists the detailed structural information of the obtained single crystal from the $\mathrm{Mg}(\mathrm{TFSI})_{2}$ in DMA-THF electrolyte.

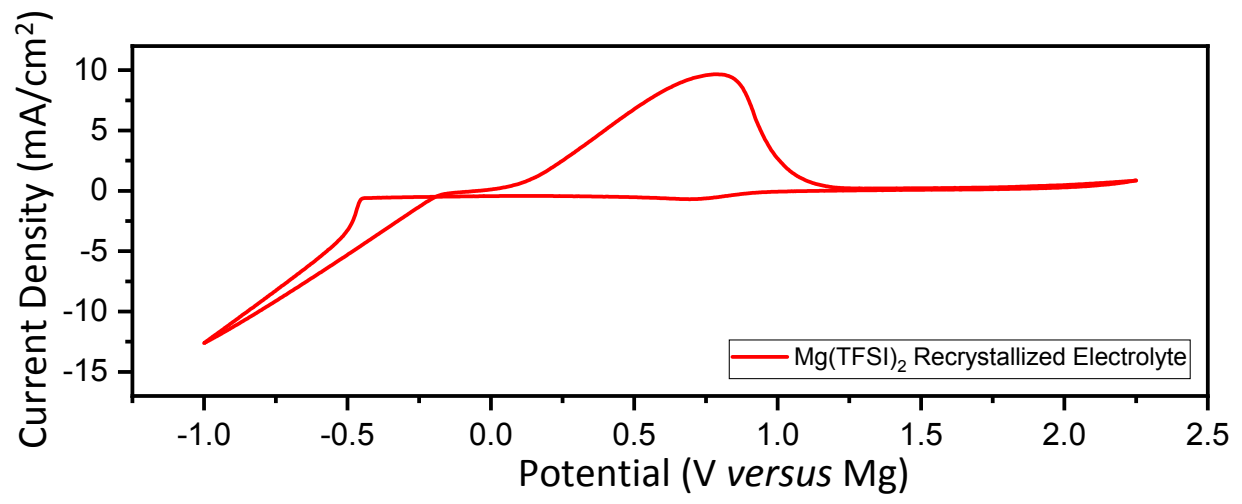

Figure S2. Cyclic voltammetry of a $0.5 \mathrm{M} \mathrm{Mg}(\mathrm{TFSI})_{2}$ in DMA-THF (2.0 M of DMA in THF) electrolyte after the recrystallized. Pt disk is used as working electrode and $\mathrm{Mg}$ polished metal is used as counter and reference electrode. Scan rate is $50 \mathrm{mV} / \mathrm{sec}$.

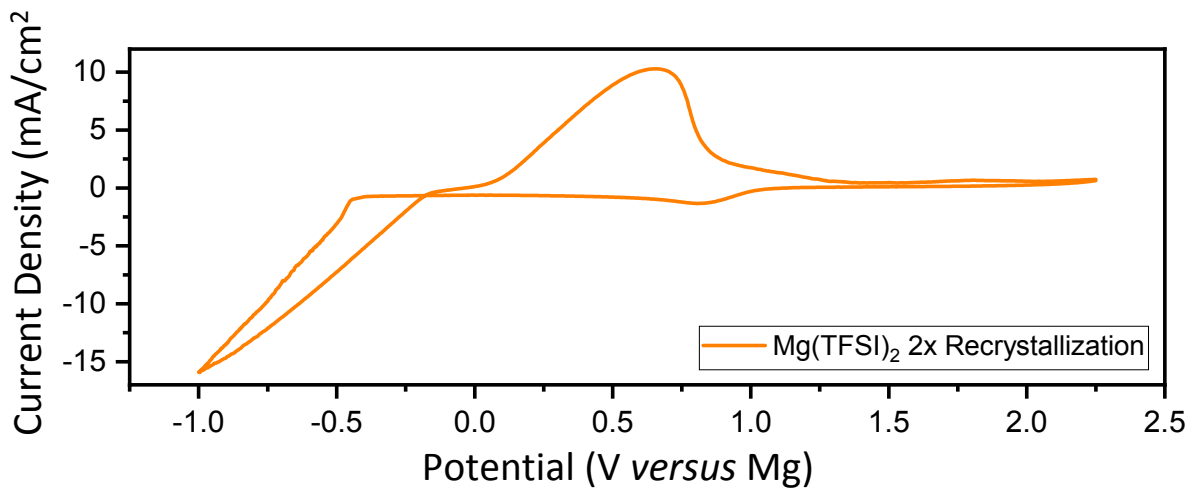

Figure S3. Cyclic voltammetry of the $0.5 \mathrm{M} \mathrm{Mg(TFSI)2} \mathrm{in} \mathrm{DMA-THF} \mathrm{(2.0} \mathrm{M} \mathrm{of} \mathrm{DMA} \mathrm{in} \mathrm{THF)}$ electrolyte after second time recrystallization. Pt disk is used as working electrode and $\mathrm{Mg}$ polished metal is used as counter and reference electrode. Scan rate is $50 \mathrm{mV} / \mathrm{sec}$.

Table S1. Detailed single crystal structural information from a $0.5 \mathrm{M} \mathrm{Mg(TFSI)2} \mathrm{in} \mathrm{DMA-THF}$ (2.0 M of DMA in THF) electrolyte 


\begin{tabular}{|c|c|}
\hline Chemical formula & $\mathrm{C}_{20} \mathrm{H}_{32} \mathrm{~F}_{12} \mathrm{MgN}_{2} \mathrm{O}_{12} \mathrm{~S}_{4}$ \\
\hline Molecular weight & 873.03 \\
\hline $\mathrm{T}(\mathrm{K})$ & 150 \\
\hline$\lambda(\AA)$ & $0 . .71073$ \\
\hline $\mathrm{a}(\AA)$ & $16.3435(16)$ \\
\hline $\mathrm{b}(\AA)$ & $13.0462(13)$ \\
\hline $\mathrm{c}(\AA)$ & $17.0892(17)$ \\
\hline$\alpha\left(^{\circ}\right)$ & 90.0 \\
\hline$\beta\left(^{\circ}\right)$ & $111.869(2)$ \\
\hline$\gamma\left({ }^{\circ}\right)$ & 90.0 \\
\hline $\mathrm{V}\left(\AA^{3}\right)$ & $3381.6(6)$ \\
\hline Space group & $\mathrm{C} 2 / \mathrm{c}$ \\
\hline $\mathrm{Z}$ & 4 \\
\hline $\mathrm{D}_{\mathrm{cal}}\left(\mathrm{g} / \mathrm{cm}^{3}\right)$ & 1.715 \\
\hline $\mathrm{U}\left(\mathrm{cm}^{-1}\right)$ & 4.25 \\
\hline $\mathrm{R} 1, w \mathrm{wR} 2(\mathrm{I}>2 \sigma(\mathrm{I}))$ & $0.0701,0.17$ \\
\hline
\end{tabular}

\section{Electrochemical properties of $\mathrm{Mg}(\mathrm{TFSI})_{2}$ in glymes without addition of the DMA co-solvent:}
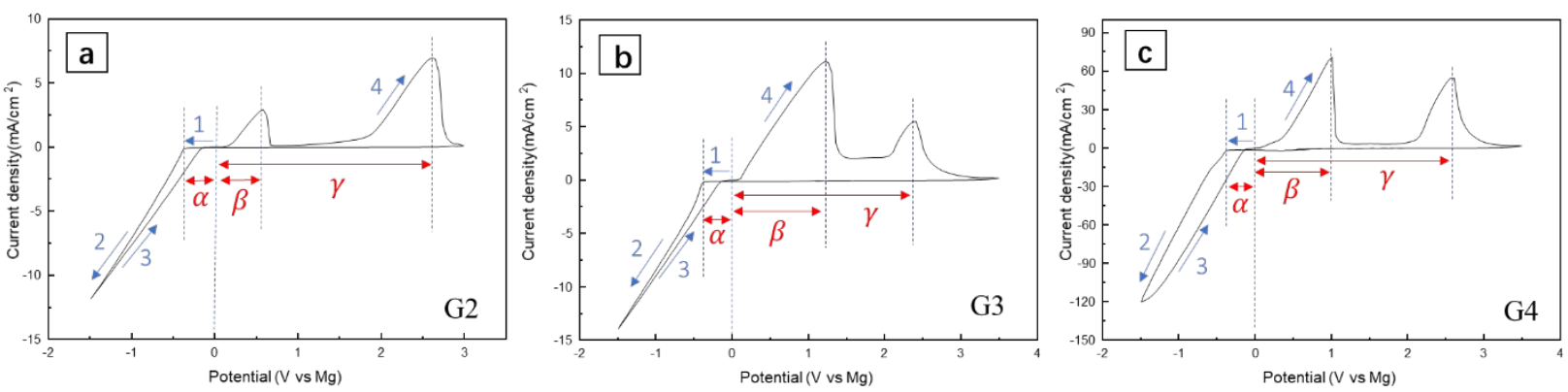

Figure S4. Cyclic voltammetry of a $0.5 \mathrm{M} \mathrm{Mg}(\mathrm{TFSI})_{2}$ in diglyme (a), triglyme (b) and tetraglyme (c) electrolyte without addition of the co-solvent of DMA. Pt disk is used as working electrode and $\mathrm{Mg}$ polished metal is used as counter and reference electrode. Scan rate is $50 \mathrm{mV} / \mathrm{sec}$.

Table S2. Detailed electrochemical overpotential information for the $\mathrm{Mg}(\mathrm{TFSI})_{2}$ in diglyme, triglyme and tetraglyme electrolytes without addition of the co-solvent of DMA.

\begin{tabular}{cccccc}
\hline solvent & \# of stripping peak & CE $(\%)$ & $\alpha(V)$ & $\beta(V)$ & $\gamma(V)$ \\
\hline DMA-THF & 1 & 56 & 0.46 & 0.72 & $\mathrm{n} / \mathrm{a}$ \\
G2 & 2 & 35 & 0.38 & 0.58 & 2.60 \\
G3 & 2 & 70 & 0.37 & 1.21 & 2.41 \\
G4 & 2 & 38 & 0.36 & 1.00 & 2.61 \\
\hline
\end{tabular}



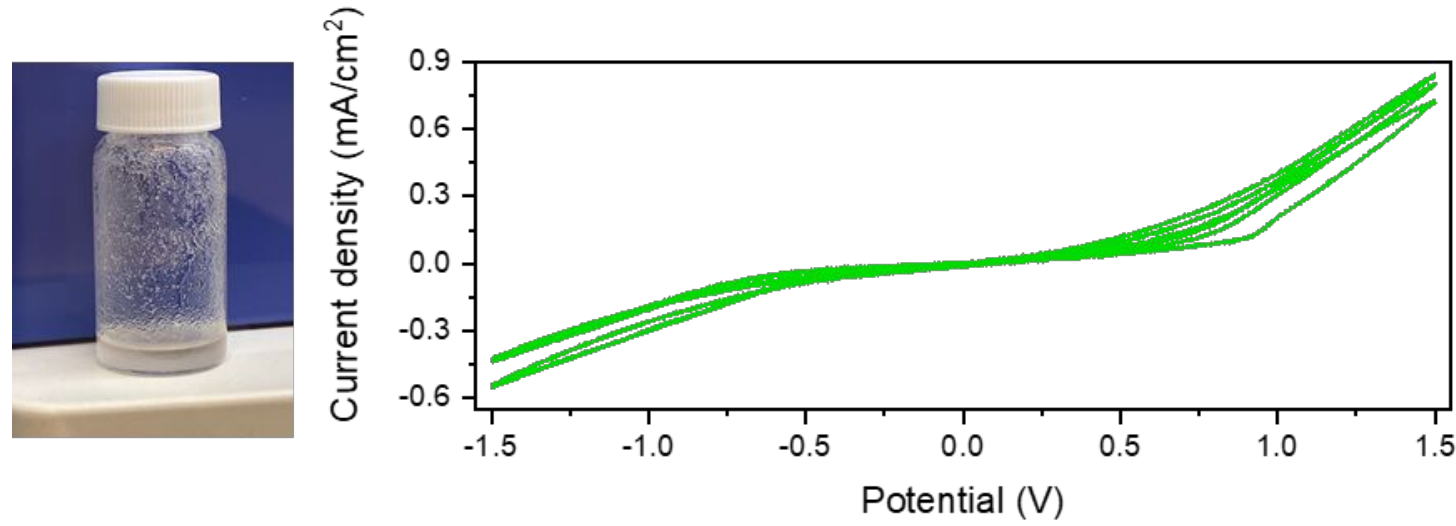

Figure S5. Left: image of a $0.5 \mathrm{M} \mathrm{Mg}(\mathrm{TFSI})_{2}$ dissolved in THF solvent; Right: corresponding CV at $50 \mathrm{mV} / \mathrm{sec}$ for the as prepared $0.5 \mathrm{M} \mathrm{Mg}(\mathrm{TFSI})_{2}$ in THF.

Table S3. Atomic-atomic distance of the crystal structure

\begin{tabular}{|c|c|c|}
\hline number $\mathrm{A}$ & number $B$ & bond length (A) \\
\hline $1 \mathrm{~S} 1$ & 02 & $1.448(3)$ \\
\hline 102 & S1 & $1.448(3)$ \\
\hline $2 \mathrm{S1}$ & 03 & $1.422(3)$ \\
\hline 203 & S1 & $1.422(3)$ \\
\hline $3 \mathrm{~S} 1$ & N1 & $1.544(2)$ \\
\hline 3 N1 & S1 & $1.544(2)$ \\
\hline $4 \mathrm{~S} 1$ & C2 & $1.824(4)$ \\
\hline $4 \mathrm{C2}$ & S1 & $1.824(4)$ \\
\hline $5 \mathrm{~S} 2$ & 04 & $1.417(3)$ \\
\hline 504 & S2 & $1.417(3)$ \\
\hline $6 \mathrm{~S} 2$ & 05 & $1.415(3)$ \\
\hline 605 & S2 & $1.415(3)$ \\
\hline $7 \mathrm{~S} 2$ & N1 & $1.589(3)$ \\
\hline $7 \mathrm{~N} 1$ & S2 & $1.589(3)$ \\
\hline $8 \mathrm{~S} 2$ & $\mathrm{C} 1$ & $1.824(4)$ \\
\hline $8 \mathrm{Cl}$ & S2 & $1.824(4)$ \\
\hline $9 \mathrm{Mg}$ & 106 & 2.0561 \\
\hline 906 & Mg1 & 2.0561 \\
\hline $10 \mathrm{M}$ & g10 & 2.074 \\
\hline 100 & $2 \mathrm{Mg}$ & 2.074 \\
\hline $11 \mathrm{M}$ & g1 0 & 2.073 \\
\hline 110 & $1 \mathrm{Mg}$ & 2.073 \\
\hline $12 \mathrm{M}$ & g10 & 2.056 \\
\hline 120 & $6 \mathrm{Mg}$ & 2.056 \\
\hline $13 \mathrm{M}$ & g10 & 2.074 \\
\hline 130 & $2 \mathrm{Mg}$ & 2.074 \\
\hline $14 M$ & g10 & 2.073 \\
\hline 140 & $1 \mathrm{Mg}$ & 2.073 \\
\hline $15 \mathrm{~F}$ & $6 \mathrm{C2}$ & $1.326(5$ \\
\hline $15 \mathrm{C}$ & $2 \mathrm{FG}$ & $1.326(5$ \\
\hline $16 \mathrm{~F}$ & $5 \mathrm{C} 2$ & $1.328(4$ \\
\hline $16 \mathrm{C}$ & $2 \mathrm{~F} 5$ & $1.328(4$ \\
\hline 170 & $6 \mathrm{C7}$ & $1.458(5)$ \\
\hline $17 \mathrm{C}$ & 706 & $1.458(5)$ \\
\hline 180 & $6 \mathrm{C} 1$ & $1.458(4$ \\
\hline $18 \mathrm{C}$ & 100 & $1.458(4$ \\
\hline $19 \mathrm{~F}$ & $4 \mathrm{C} 2$ & $1.329(3$ \\
\hline $19 \mathrm{C}$ & $2 \mathrm{~F} 4$ & $1.329(3$ \\
\hline $20 \mathrm{~F}$ & $1 \mathrm{C} 1$ & $1.312(5$ \\
\hline $20 \mathrm{C}$ & $1 \mathrm{~F} 1$ & $1.312(5$ \\
\hline $21 \mathrm{~F}$ & $3 \mathrm{C} 1$ & $1.327(4$ \\
\hline $21 \mathrm{C}$ & $1 \mathrm{~F} 3$ & $1.327(4$ \\
\hline 220 & $1 \mathrm{C} 6$ & $1.445(5)$ \\
\hline $22 \mathrm{C}$ & 601 & $1.445(5)$ \\
\hline 230 & $1 \mathrm{C} 3$ & $1.432(8)$ \\
\hline $23 \mathrm{C}$ & 301 & $1.432(8)$ \\
\hline $24 \mathrm{~F}$ & $2 \mathrm{C} 1$ & $1.319(5$ \\
\hline $24 \mathrm{C}$ & $1 \mathrm{~F} 2$ & $1.319(5$ \\
\hline $25 \mathrm{C}$ & $7 \mathrm{H} 7$ & 0.97 \\
\hline $25 \mathrm{H}$ & $7 A C$ & 0.97 \\
\hline $26 \mathrm{C}$ & $7 \mathrm{H} 7$ & 0.969 \\
\hline $26 \mathrm{H}$ & $7 B C$ & 0.969 \\
\hline $27 \mathrm{C}$ & $7 C 8$ & $1.494(5)$ \\
\hline $27 \mathrm{C}$ & $8 \mathrm{C7}$ & $1.494(5)$ \\
\hline $28 \mathrm{C}$ & $10 \mathrm{H}$ & 0.96 \\
\hline $28 \mathrm{H}$ & $10 \mathrm{~A}$ & 0.96 \\
\hline $29 \mathrm{C}$ & $10 \mathrm{H}$ & 0.97 \\
\hline $29 \mathrm{H}$ & $10 B$ & 0.97 \\
\hline $30 \mathrm{C}$ & $10 \mathrm{C}$ & $1.500(5$ \\
\hline $30 \mathrm{C}$ & $9 \mathrm{C} 1$ & $1.500(5$ \\
\hline $31 \mathrm{C}$ & $6 \mathrm{H} 6$ & 0.97 \\
\hline
\end{tabular}

\begin{tabular}{|c|c|c|}
\hline $31 \mathrm{C}$ & $6 \mathrm{H} 6$ & 0.97 \\
\hline $31 \mathrm{H}$ & $6 A C$ & 0.97 \\
\hline $32 \mathrm{C}$ & $6 \mathrm{H} 6$ & 0.971 \\
\hline $32 \mathrm{H}$ & $6 B C$ & 0.971 \\
\hline $33 \mathrm{C}$ & $6 \mathrm{C5}$ & $1.500(5)$ \\
\hline $33 \mathrm{C}$ & $5 \mathrm{C6}$ & $1.500(5)$ \\
\hline $34 \mathrm{C}$ & $8 \mathrm{H} 8$ & 0.971 \\
\hline $34 \mathrm{H}$ & $8 \mathrm{AC}$ & 0.971 \\
\hline $35 \mathrm{C}$ & $8 \mathrm{H} 8$ & 0.969 \\
\hline $35 \mathrm{H}$ & $8 B C$ & 0.969 \\
\hline $36 \mathrm{C}$ & $8 \mathrm{Cg}$ & $1.520(5)$ \\
\hline $36 \mathrm{C}$ & $9 \mathrm{C} 8$ & $1.520(5)$ \\
\hline $37 \mathrm{C}$ & $9 \mathrm{H} 9$ & 0.969 \\
\hline $37 \mathrm{H}$ & $9 A C$ & 0.969 \\
\hline $38 \mathrm{C}$ & $9 \mathrm{H} 9$ & 0.97 \\
\hline $38 \mathrm{H}$ & $9 B C$ & 0.97 \\
\hline $39 \mathrm{C}$ & $4 \mathrm{H} 4$ & 0.97 \\
\hline $39 \mathrm{H}$ & $4 A C$ & 0.97 \\
\hline $40 \mathrm{C}$ & $4 \mathrm{H} 4$ & 0.969 \\
\hline $40 \mathrm{H}$ & $4 B C$ & 0.969 \\
\hline $41 \mathrm{C}$ & $4 C 5$ & $1.523(7)$ \\
\hline $41 \mathrm{C}$ & $5 C 4$ & $1.523(7)$ \\
\hline $42 \mathrm{C}$ & $4 C 3$ & $1.407(9)$ \\
\hline $42 \mathrm{C}$ & $3 C 4$ & $1.407(9)$ \\
\hline $43 \mathrm{C}$ & $5 \mathrm{H} 5$ & 0.97 \\
\hline $43 \mathrm{H}$ & $5 A C$ & 0.97 \\
\hline $44 \mathrm{C}$ & $5 \mathrm{H} 5$ & 0.969 \\
\hline $44 \mathrm{H}$ & $5 B C$ & 0.969 \\
\hline $45 \mathrm{C}$ & $3 \mathrm{H} 3$ & 0.971 \\
\hline $45 \mathrm{H}$ & $3 A C$ & 0.971 \\
\hline $46 C$ & $3 \mathrm{H} 3$ & 0.97 \\
\hline $46 \mathrm{H}$ & $3 B C$ & 0.97 \\
\hline $47 \mathrm{~S}$ & 102 & $1.448(3$ \\
\hline 470 & $2 \mathrm{~S} 1$ & $1.448(3$ \\
\hline $48 \mathrm{~S}$ & 103 & $1.422(3$ \\
\hline 480 & $3 \mathrm{~S} 1$ & $1.422(3$ \\
\hline $49 \mathrm{~S}$ & $1 \mathrm{~N} 1$ & $1.544(2$ \\
\hline $49 \mathrm{~N}$ & $1 \mathrm{~S} 1$ & $1.544(2$ \\
\hline $50 \mathrm{~S}$ & $1 \mathrm{C} 2$ & $1.824(4$ \\
\hline $50 \mathrm{C}$ & $2 \mathrm{~S} 1$ & $1.824(4$ \\
\hline $51 \mathrm{~S}$ & 204 & $1.417(3$ \\
\hline 510 & $4 \mathrm{~S} 2$ & $1.417(3$ \\
\hline $52 \mathrm{~S}$ & 205 & $1.415(3$ \\
\hline 520 & $5 \mathrm{~S} 2$ & $1.415(3$ \\
\hline $53 \mathrm{~S}$ & $2 \mathrm{~N} 1$ & $1.589(3$ \\
\hline $53 \mathrm{~N}$ & $1 \mathrm{~S} 2$ & $1.589(3$ \\
\hline $54 \mathrm{~S}$ & $2 \mathrm{C} 1$ & $1.824(4$ \\
\hline $54 \mathrm{C}$ & $1 \mathrm{~S} 2$ & $1.824(4$ \\
\hline $55 \mathrm{~F}$ & $6 \mathrm{C} 2$ & $1.326(5$ \\
\hline $55 \mathrm{C}$ & $2 \mathrm{~F} 6$ & $1.326(5$ \\
\hline $56 \mathrm{~F}$ & $5 \mathrm{C} 2$ & $1.328(4$ \\
\hline $56 \mathrm{C}$ & $2 \mathrm{~F} 5$ & $1.328(4$ \\
\hline 570 & $6 \mathrm{C7}$ & $1.458(5)$ \\
\hline $57 \mathrm{C}$ & 706 & $1.458(5)$ \\
\hline 580 & $6 \mathrm{C} 1$ & $1.458(4$ \\
\hline $58 \mathrm{C}$ & 100 & $1.458(4$ \\
\hline $59 \mathrm{~F}$ & $4 \mathrm{C} 2$ & $1.329(3$ \\
\hline $59 \mathrm{C}$ & $2 \mathrm{~F} 4$ & $1.329(3$ \\
\hline $60 \mathrm{~F}$ & $1 \mathrm{C} 1$ & $1.312(5$ \\
\hline $60 \mathrm{C}$ & $1 \mathrm{~F} 1$ & $1.312(5$ \\
\hline
\end{tabular}

\begin{tabular}{|c|c|c|}
\hline $60 \mathrm{~F}$ & $1 \mathrm{C} 1$ & $1.312(5$ \\
\hline $60 \mathrm{C}$ & $1 \mathrm{~F} 1$ & $1.312(5$ \\
\hline $61 \mathrm{~F}$ & $3 \mathrm{C} 1$ & $1.327(4$ \\
\hline $61 \mathrm{C}$ & $1 \mathrm{~F} 3$ & $1.327(4$ \\
\hline 620 & $1 \mathrm{C} 6$ & $1.445(5)$ \\
\hline $62 C$ & 601 & $1.445(5)$ \\
\hline 630 & $1 \mathrm{C} 3$ & $1.432(8)$ \\
\hline $63 C$ & 301 & $1.432(8)$ \\
\hline $64 \mathrm{~F}$ & $2 \mathrm{C} 1$ & $1.319(5$ \\
\hline $64 C$ & $1 \mathrm{~F} 2$ & $1.319(5$ \\
\hline $65 \mathrm{C}$ & $7 \mathrm{H} 7$ & 0.97 \\
\hline $65 \mathrm{H}$ & $7 A C$ & 0.97 \\
\hline $66 \mathrm{C}$ & $7 \mathrm{H} 7$ & 0.969 \\
\hline $66 \mathrm{H}$ & $7 B C$ & 0.969 \\
\hline $67 C$ & $7 \mathrm{C} 8$ & $1.494(5)$ \\
\hline $67 C$ & $8 \mathrm{C7}$ & $1.494(5)$ \\
\hline $68 \mathrm{C}$ & $10 \mathrm{H}$ & 0.96 \\
\hline $68 \mathrm{H}$ & $10 \mathrm{~A}$ & 0.96 \\
\hline $69 \mathrm{C}$ & $10 \mathrm{H}$ & 0.97 \\
\hline $69 \mathrm{H}$ & $10 B$ & 0.97 \\
\hline $70 \mathrm{C}$ & $10 \mathrm{C}$ & $1.500(5$ \\
\hline $70 \mathrm{C}$ & $9 \mathrm{C} 1$ & $1.500(5$ \\
\hline $71 \mathrm{C}$ & $6 \mathrm{H} 6$ & 0.97 \\
\hline $71 \mathrm{H}$ & $6 A C$ & 0.97 \\
\hline $72 \mathrm{C}$ & $6 \mathrm{H} 6$ & 0.971 \\
\hline $72 \mathrm{H}$ & $6 B C$ & 0.971 \\
\hline $73 C$ & $6 \mathrm{C5}$ & $1.500(5)$ \\
\hline $73 \mathrm{C}$ & $5 \mathrm{C} 6$ & $1.500(5)$ \\
\hline $74 \mathrm{C}$ & $8 \mathrm{H} 8$ & 0.971 \\
\hline $74 \mathrm{H}$ & $8 \mathrm{AC}$ & 0.971 \\
\hline $75 \mathrm{C}$ & $8 \mathrm{H} 8$ & 0.969 \\
\hline $75 \mathrm{H}$ & $8 B C$ & 0.969 \\
\hline $76 \mathrm{C}$ & $8 \mathrm{C9}$ & $1.520(5)$ \\
\hline $76 \mathrm{C}$ & $9 \mathrm{C} 8$ & $1.520(5)$ \\
\hline $77 \mathrm{C}$ & $9 \mathrm{H} 9$ & 0.969 \\
\hline $77 \mathrm{H}$ & $9 A C$ & 0.969 \\
\hline $78 \mathrm{C}$ & $9 \mathrm{H} 9$ & 0.97 \\
\hline $78 \mathrm{H}$ & $9 B C$ & 0.97 \\
\hline $79 \mathrm{C}$ & $4 \mathrm{H} 4$ & 0.97 \\
\hline $79 \mathrm{H}$ & $4 A C$ & 0.97 \\
\hline $80 \mathrm{C}$ & $4 \mathrm{H} 4$ & 0.969 \\
\hline $80 \mathrm{H}$ & $4 B C$ & 0.969 \\
\hline $81 \mathrm{C}$ & $4 \mathrm{C5}$ & $1.523(7)$ \\
\hline $81 \mathrm{C}$ & $5 \mathrm{C4}$ & $1.523(7)$ \\
\hline $82 \mathrm{C}$ & $4 \mathrm{C3}$ & $1.407(9)$ \\
\hline $82 \mathrm{C}$ & $3 C 4$ & $1.407(9)$ \\
\hline $83 \mathrm{C}$ & $5 \mathrm{H} 5$ & 0.97 \\
\hline $83 \mathrm{H}$ & $5 A C$ & 0.97 \\
\hline $84 \mathrm{C}$ & $5 \mathrm{H} 5$ & 0.969 \\
\hline $84 \mathrm{H}$ & $5 B C$ & 0.969 \\
\hline $85 \mathrm{C}$ & $3 \mathrm{H} 3$ & 0.971 \\
\hline $85 \mathrm{H}$ & $3 A C$ & 0.971 \\
\hline $86 \mathrm{C}$ & $3 \mathrm{H} 3$ & 0.97 \\
\hline $86 \mathrm{H}$ & $3 B C$ & 0.97 \\
\hline
\end{tabular}




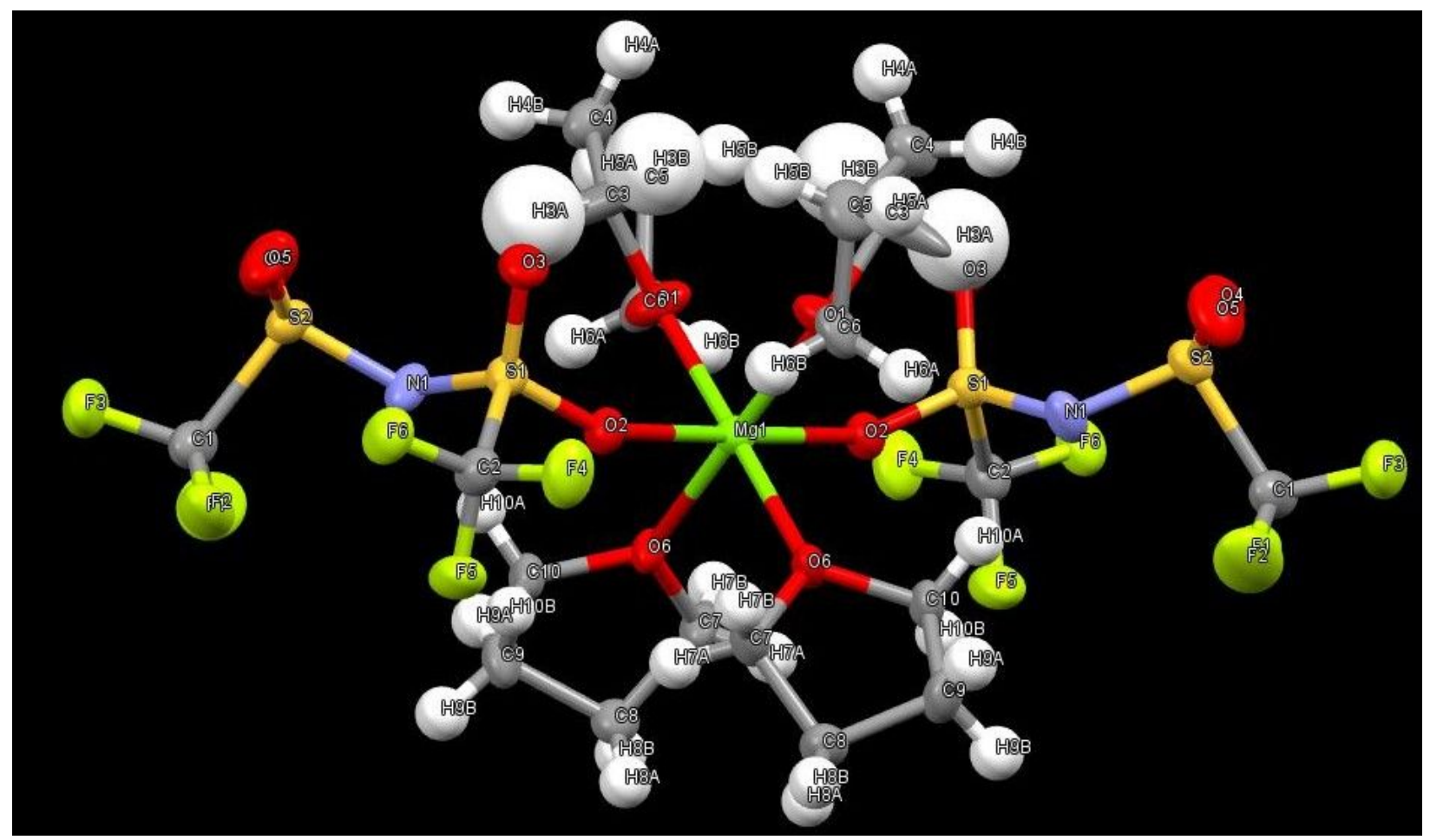

Figure S6. An enlarged single crystal structure of the $\mathrm{Mg}(\mathrm{TFSI})_{2}(\mathrm{THF})_{4}$ electrolyte with labeled atomic numbers in accordance with atomic-atomic distance information from Table S3. Displacement ellipsoid representation (50\% probability level). 